\title{
Image Enhancement Techniques: A Review
}

\author{
Abhinav Bansal ${ }^{1}$, Navdeep Singh ${ }^{2}$ \\ ${ }^{1}$ Department of Computer Science and Engineering, Punjabi University, Patiala, Punjab , India ${ }^{2}$ Department of Computer \\ Science and Engineering, Punjabi University, Patiala, Punjab, India \\ 1abhinavbansal2090@gmail.com ${ }^{2}$ navdeepsony@gmail.com
}

\begin{abstract}
Image enhancement is the process of noise removal from images, brighten or sharpen the images to clearly identifying the key features of the image. To optimize the image, various contrast enhancement techniques are used. In this paper, some image enhancement techniques have been evaluated.
\end{abstract}

Keyword- Histogram, Image, Contrast, Perceptual color transfer, Attenuation.

\section{INTRODUCTION}

Image enhancement is required to visualize every feature of an image. Images that are captured in darkness contains poor visibility and contrast of the image is low. Image quality can be increased through various techniques.

\section{LITERATURE SURVEY}

In this paper, analysis of various image enhancement techniques has been done.

\section{A. Image Contrast Enhancement using Color and Depth Algorithms [1]}

Method is proposed by Seung-Won Jung[1].

In this paper, Gaussian mixture model (GMM) method is used which is effective in contast enhancement.

Histogram of the color image modifies using the histogram of depth image.Histogram of the color image can be represented by transforming the RGB space to hue-saturation-intensity space(HSI) space. Hence, histogram modification is applied to intensity channels. Resultant image is obtained by transforming the color space back to RGB space[1].

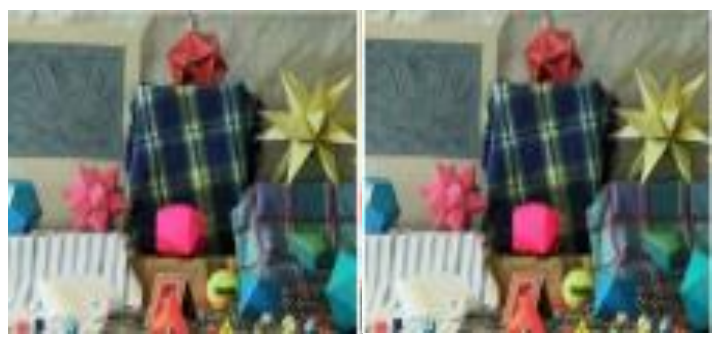

Fig 1.a) Input image [1] b) Enhanced image [1]

\section{B. Histogram-Based Locality-Preserving Contrast Enhancement [2]}

In this method is proposed by Jeyong Shin,et al.[2]

a. Locality condition is defined in image histogram by using the intensity level. The purpose of the locality condition is to re-alize a local Contrast Enhancement [2]

b. Optimization Problem Formulation

To calculate $\mathrm{x}$, an optimization problem is formed in the context of locality conditions over the entire intensity range [2].

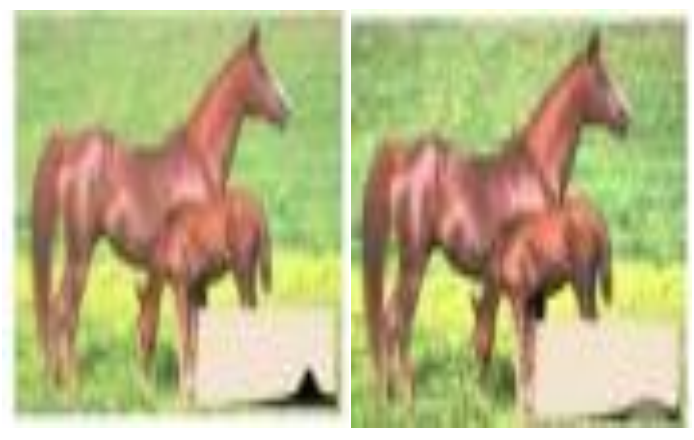

Fig 2. a)Input image[2] b)Enhanced image[2]

\section{A Low-Light Image Enhancement Method For Both Denoising And Contrast Enlarging[4]}

Method is proposed by Lin Li,et al[4]

In this paper, the proposed method is enlightened by dehazing-based algorithm and unsharp masking algorithm [4].

There are two stages in this method: superpixel based adaptive denoising and the second stage is luminance adaptive contrast enhancement.

a. Superpixel level adaptive denoising

Denoising filter blurs the texture details. So its desired that strength of denoising filter can be adaptive according to the local feature of image patch[4]. Strong filter is preferred for high noise 
visibility and weak filter is preferred for low noise visibility [4].

\section{b. Luminance adaptive contrast enhancement}

Haze removal method is used to enhance the contrast of the image [4].

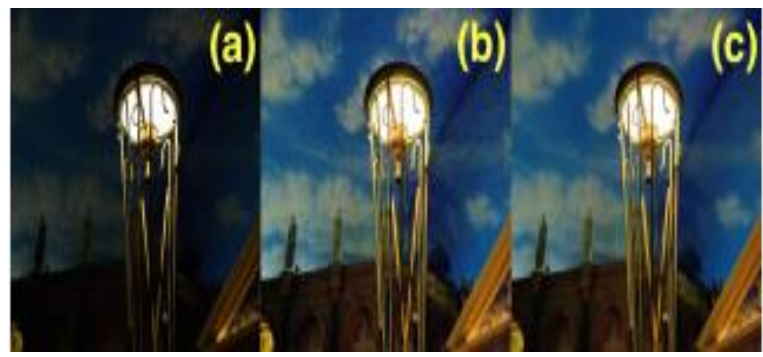

Fig 3. a) Input image[4] b) Contrast enhanced Image without noise reduction[4] c) The final Enhanced image[4]

\section{A Combined effect of local and global method for contrast image enhancement [3]}

Method is proposed by Sampada S Pathak [3]

In this paper, this technique is used on $2 \mathrm{D}$ histogram.2D histogram is made up of RGB values.

Global contrast stretching method is used to perform global contrast image enhancement.

Local contrast image enhancement is used to perform local contrast stretching method.

The Proposed method is used to preserve the brightness [3].

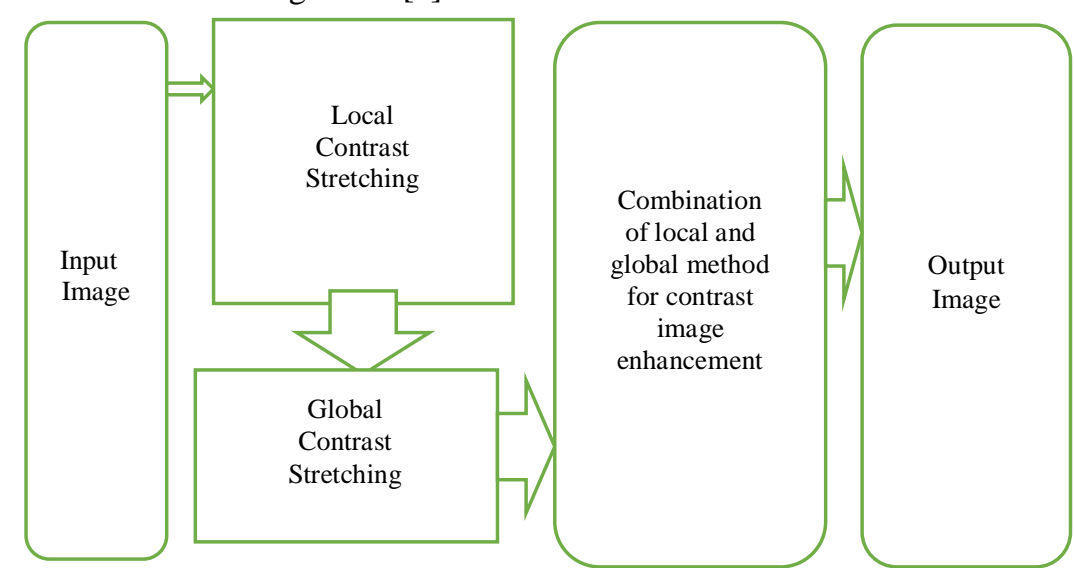

Fig 4. Flowchart [3]

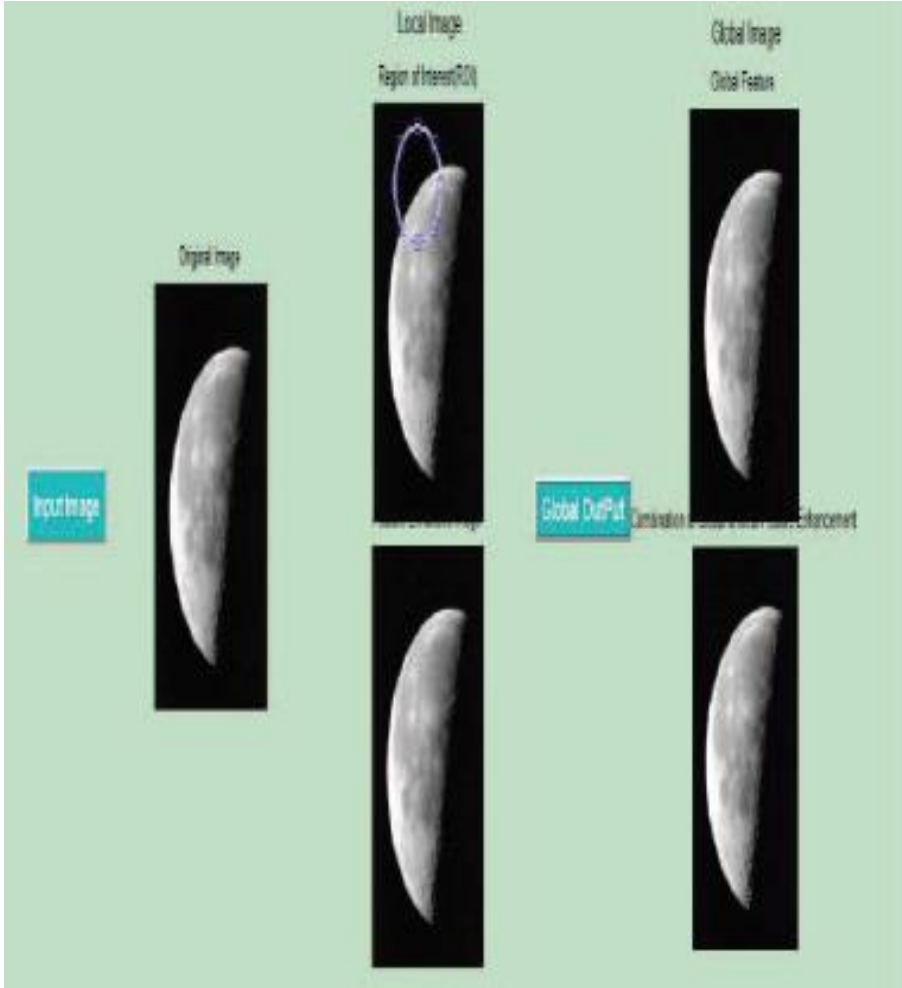

Fig 5. GUI of combination of local and global contrast image enhancement method[3]

\section{E. Dark Image Enhancement Using Perceptual Color Transfer [9]}

Method is proposed by Jonathan Cepeda-Negrete et al. [9]

In this paper, Perceptual color transfer (PCT) technique to transform a dark image into a lightened scene [9].A classical color transfer technique is used to perform the transformation of dark image into lighten image[9].

Details of methodology explain in details below.

a. Color transfer using first order statistics

In this method, there are two measures calculated in the image i.e. global mean and standard deviation. The purpose of this method is to transform an input image into another image which has similar look to that of the reference image [9].

b. Color transfer in the RLAB perceptual color space

In this methodology, The usage of color transfer is explore for complete dark images

C. Assessment of the color transfer 
In this methodology, metric is used for evaluating the quality of the image, similarity between the outcome and the target image is calculated. The comparison measure uses the histograms of the images [9].

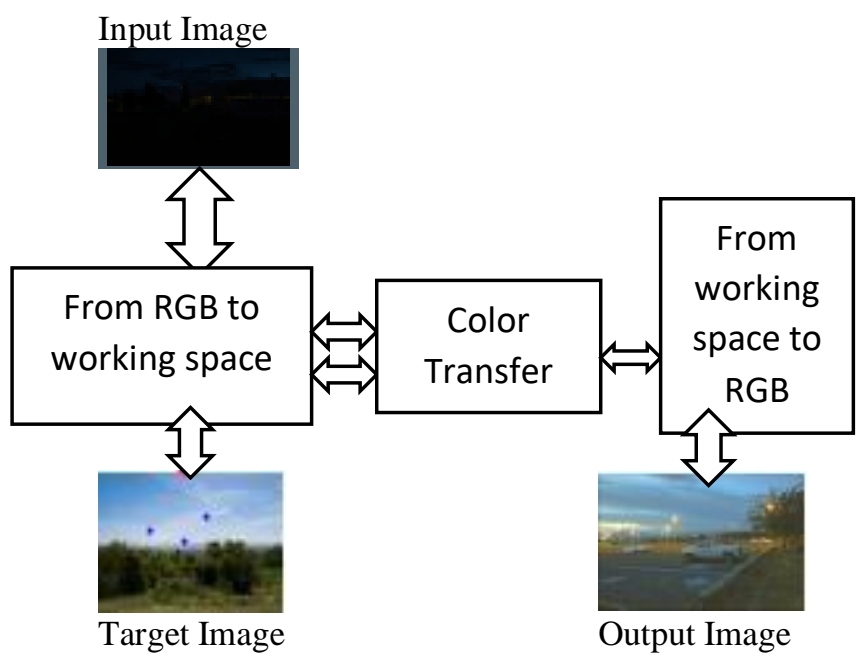

Fig 6. Color transfer procedure upon a dark image [9]

\section{F. Nighttime Driving Safety Improvement via Image Enhancement for Driver Face Detection [10]}

Method is proposed by Jianhao Shen et al.[10]

In this paper the author proposed proposes an adaptive attenuation quantification retinex (AAQR) method to enhance the features of images captured at night time. There are three steps in this method: attenuation restriction, attenuation prediction, and adaptive quantification [10].

Details of methodology explain in details below.

\section{a. Original Retinex Model}

The Retinex theory has the ability to enhance features of images taken under complicated lighting conditions. The most conventional Retinex model is processed in logarithm space because it decreases complexity [10].

\section{b. Adaptive Attenuation Quantification} Retinex (AAQR)

AAQR is the illumination- robust imageprocessing method. This method is proposed to enhance the features of drivers' face image captured at night so that face detection can be improved [10]. AAQR consists of three steps i.e. attenuation restriction, attenuation prediction, and adaptive quantification.

\section{Attenuation Restriction}

In this step, mean and variance of the pixels is calculated to compute the brightness range of an image.

\section{Attenuation Prediction}

The Retinex image of the logarithmic domain $\mathrm{iR} \log (\mathrm{x} ; \mathrm{y})$ is computed as $\mathrm{iR} \log (\mathrm{x} ; \mathrm{y}) \mathrm{D} \log \mathrm{Ri}(\mathrm{x}$; $y) . i R \log (x ; y)$ is obtained by subtracting the lowpass filtered image from the original image in the logarithmic domain.

\section{Adaptive Quantification}

In this step, pixels of attenuation area should be assigned new gray scales to ignore negative values in quantization process [10].

\section{c. Face Detection}

This method is used to evaluate the performance of AAQR algorithm. There are 3 phases face detection, face alignment and face validation. [10]

\section{Face Detection}

Object's appearance and shape can be narrated through gradient or directional density distribution.[10]

\section{Face Alignment}

In this phase, drivers can capture the images by facing the camera at different positions. Thus, captured Images were re-adjusted to improve the robustness. This includes two steps: face landmark estimation and affine transformation. [10]

i) Face landmark estimation

A group of regression trees was an effective algorithm to find critical characteristics by marking 68 features in a face image [10]. It covers the details of eyes, nose, mouth, shape of a face, etc.[10]

ii) Affine transformation

Affine transformation is a linear transformation from a 2D coordinate to another 2D coordinate when the picture are kept straight and parallel[10]. 


\section{Face Validation}

In this phase, minimum distance is calculated by computing the Euclidean distances between the input image and the images in the database name it as the detected face. The probability of the AAQR enhanced image to be recognized as a human face is indicated by confidence number output from the classifier [10].

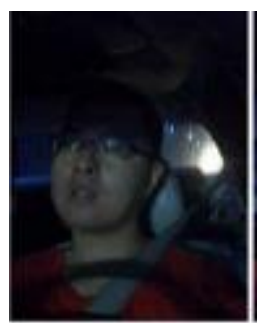

(a)

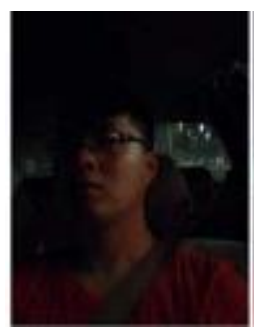

(c)

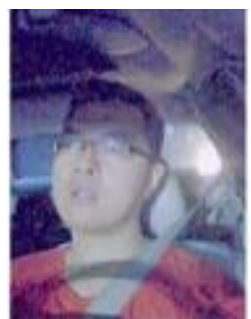

(b)

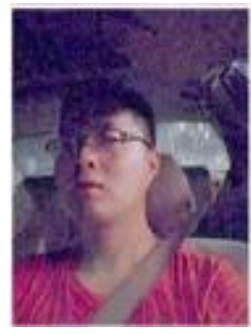

(d)
Fig 7. a) Up-Down original image [10] b) Updown result image using AAQR [10] c) Left-Right original image[10] d) Left-Right result image using AAQR[10].

\section{G. Contrast Enhancement of Microscopy Images Using Image Phase Information [11].}

Method is proposed by Serdar Cakir et al. [11]

In this paper, a technique is proposed by using the image phase information to increase the weight of frequencies where phase variations occur [11]. Mostly images captured naturally are generally low-pass by nature. Expressly, spectral energy is very dense at low frequencies compared to high frequencies in natural images. Sharp transitions and highly structured textures are contributed to high frequency components of an image. [11]

In the proposed image enhancement algorithm, the image phase is taken into consideration to emphasize high frequency components. [11]
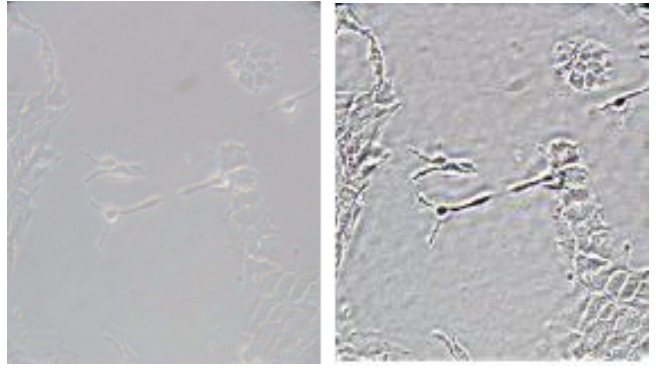

Fig 8 . a) Original image [11] b) Result image [11]

\section{CONCLUSION}

In this paper, various contrast enhancement techniques are analysed. It has been observed that all the techniques are effective in contrast enhancement. There are various algorithms used such as Gaussian mixture model (GMM) method, dehazing-based algorithm, global contrast stretching method etc.

\section{REFERENCES}

[1] Seung-Won Jung et al," Image Contrast Enhancement using Color and Depth Algorithms", IEEE Signal Processing Letters, VOL. 21, NO. 4, April 2014.

[2] Jeyong Shin ,Rae-Hong Park et al," HistogramBased Locality-Preserving Contrast Enhancement", IEEE Signal Processing Letters, VOL. 22, NO. 9, September 2015.

[3] Sampada S Pathak, Prashant Dahiwale, Ganesh Padole et al," A Combined effect of local and global method for contrast image enhancement", 2015 IEEE International Conference on Engineering and Technology (ICETECH), 20th March 2015, Coimbatore, TN, India.

[4] Lin Li, Ronggang Wang,Wenmin Wang, Wen Gao et al," A Low-Light Image Enhancement Method For Both Denoising And Contrast Enlarging",

[5] X. Dong, G. Wang, Y. Pang, W. Li, J. Wen, W. Meng, and Y. Lu, "Fast efficient algorithm for enhancement of low lighting video," in Multimedia and Expo (ICME),2011 IEEE International Conference on. IEEE, 2011, pp. 1-6.

[6] G. Deng, "A generalized unsharp masking algorithm, "Image Processing, IEEE Transactions on, vol. 20, no. 5,pp. 1249-1261, 2011.

[7] G. Deng, "A generalized unsharp masking algorithm, "Image Processing, IEEE Transactions on, vol. 20, no. 5,pp. 1249-1261, 2011. 
[8] K. He, J. Sun, and X. Tang, "Single image haze removal using dark channel prior," Pattern Analysis and Machine Intelligence(PAMI), IEEE Transactions on, vol. 33, no.12, pp. 2341-2353, 2011.

[9] J. Cepeda - Negrete et al, "Dark Image Enhancement Using Perceptual Color Transfer", VOL. 6, 2018, IEEE Access.

[10]Jianhao Shen et al," Nighttime Driving Safety Improvement via Image Enhancement for Driver Face Detection",VOL. 6,2018,IEEE Access.

[11] Sedar Cakir et al.," Contrast Enhancement of Microscopy Images Using Image Phase Information", VOL. 6, 2018, IEEE Access .

[12] E. H. Land and J. J. McCann, "Lightness and Retinex theory,' J. Opt. Soc.

Amer., vol. 61, no. 1, pp. 1-11, 1971.

[13] V. Kazemi and J. Sullivan, "One millisecond face alignment with an ensemble of regression trees,' in Proc. IEEE CVPR, Jun. 2014, pp. 1867-1874. 